\title{
Admission Patterns and Outcomes in an Adult Intensive Care Unit in Medical Patients in Karachi
}

\author{
Teena Rajput*, Fasiha Sohail, Sumera Nawaz Qabulio*, Muhammad Fahad Zakir and Misbah Younus Soomro \\ Medicine Department, Ziauddin University and Hospital, Pakistan
}

Submission: June 22, 2018; Published: August 07, 2018

*Corresponding author: Teena Rajput, Senior Resident Medical Officer, Department of Cirtical Care, Ziauddin University and Hospital, Karachi, Pakistan, Tel: +92-331-3229190; Email: drteena_sw31@outlook.com

Dr. Sumera Nawaz, Registar, Department of Medicine, Ziauddin Hospital, Karachi, Pakistan, Tel:+92-0336-2092118, Email: sumeraqabulio@gmail.com

Abstract

Aims: To Review the outcome of adult admitted patients in Intensive Care Unit (ICU) in Karachi Methods: Retrospective study, clinical diagnosis, management and response of adult patients in ICU of KDLB Ziauddin hospital Karachi from January 2017 to June 2017 were studied.

Results: 241 adults were admitted to the ICU with male to female ratio of $51 \%$ to $49 \%$. Diagnoses included. Hepatic diseases $12.9 \%$, CCF and Pulmonary edema $6.2 \%$, Cellulitis $0.8 \%$, CVA $11.6 \%$, Hypertensive Crisis $4.1 \%$, Arrhythmias $0.8 \%$, Diabetes with complication $2.5 \%$, Carcinoma $0.4 \%, 2.1 \%$ Tb with complication, Respiratory issues 9.5\%, ACS 5.4\%, Kidney diseases 5.4\%, Sepsis 10.4\%, GI Bleed 3.3\%, Heat stroke $0.4 \%$, CNS Infection7.9\%, Poisoning 3.3\%, Shock 1.2\%, AGE with complication 5.8\%, Surgical and Gynecological issues were 5.4\% and $0.4 \%$. Out of 241 admitted adult 39(\%), died 16(\%), left against medical advice 146(\%), were improved and subsequently discharged. Forty (\%) become better and shifted to ward and (\%) received mechanical ventilation.

Conclusion: Most common reason of admission found to be Hepatic, central nervous system respiratory and infectious disease. Patients on mechanical ventilation with sepsis seemed with higher mortality rates. Therefore, these patients require early referral and timely management for better outcome and early recovery.

Keywords: Critical Care; ICU; Intensive Care

\section{Introduction}

Critical care has been characterized as: administer to patients who have possibly recoverable conditions who can profit by more point by point perception (with or without intrusive treatment) than can be given securely in a conventional ward. The cuttingedge idea of the emergency unit to a great extent developed from crafted by Larsen 5 amid the polio scourge in Copenhagen in 1952. He demonstrated that passing's from respiratory disappointment drastically diminished from $87 \%$ to $40 \%$ with the change from cuirasse ventilation (the 'iron lung') to manual positive weight ventilation through a bound tracheotomy tubes [1]. Within the half century ( 50 years) critical care medicine has spread to entire world [2]. Critical care is a sweeping strength with practically boundless limits. Although the organization of critical care services varies from country to country. Like every country in Pakistan there is Critical care society too [3]. The general public keeps up the right care, right now.

In the context of Pakistan, the availability of ICU is largely limited to major cities in the country. In recent decade an improved future, a greater mass of maturing and consistently developing overhauls in the movement of medicinal services framework has extended the enthusiasm for critical mind organizations. In any hospital critical care team consist of critical care, internvists, residents, nurse practitioners, ICU, technicians, physician, assistants, physician specialists, primary care, physicians, respiratory therapists, other professionals, also referred to as the multi professional team model. Multi professional team optimizes care for patients, give the educational knowledge to the patient's family attendants for their future healthcare, improves conditions for healthcare providers, and boosts the financial performance of the hospital Adult Intensive care admission vary within city to city of same country too depending upon the prevalence and epidemic of the disease in that particular city [4].

One should be aware of the prevalent conditions and set up the facilities and prepare treatment. In our ICU more than 400 patients admitted annually in different age group of either sex with multiple diseases. About $80 \%$ Patients got admitted via e.r., $15 \%$ from different wards and $5 \%$ from operation theatre. Often these patients arrived late with multiple complications that are leading to increased mortality and morbidity despite the best available therapy [5]. About one third of hospital mortality occurs in critically ill patients inside Intensive Care Unit (ICU). Along with this Poverty, illiteracy, lack of proper transportation facilities and lack of resources further adds to the challenge in management. These facts are highlighting the strong necessity to strengthen the existing health care system and develop facilities for proper 
transportation and treatment of critically ill patients in private as well as government setup [6]. Only few hours delay in transferring a critically ill patient from emergency to intensive care unit is independently associated with poor outcome at discharge [7].

Study carried out at Lagos University Teaching Hospital (LUTH) showed need to focus and improve system of health evaluation. Previously several studies had conducted regarding pattern and outcomes in ICU [8]. In tertiary referral hospital of Tanzia male comprised the major proportion of study and overall mortality rate was $41.4 \%$ [9]. In Netherland from 1997-2001 study showed Hospital mortality for ICU-admitted patients in the NICE registration was $12.9 \%$ [10].

\section{Materials and Methods}

This is a retrospective review of all medical patients admitted into the general ICU at campus of Ziauddin university in Karachi from January 2017 to June 2017. Data is collected from the ICU admission and discharge registers, and data analysis was done using EXCEL VERSION 2007.ICU equipped with central oxygen supply and suction lines, infusion pumps, conventional mechanical ventilator, on invasive ventilation, portable X-ray machine, bedside ultrasonography, bedside upper gastrointestinal endoscopy defibrillators, patient care monitors and ECG machine. However, we do not have facilities for intra-cranial pressure monitoring, bedside

echocardiography, plasmapheresis, dialysis. There are one resident doctor and one resident medical officer on duty supported by 3 trained nurses. The doctors on-duty are also responsible for looking after the 10 bedded adult ICU under the supervision of 2 senior consultants including ICU internist and physician (Figure 1).

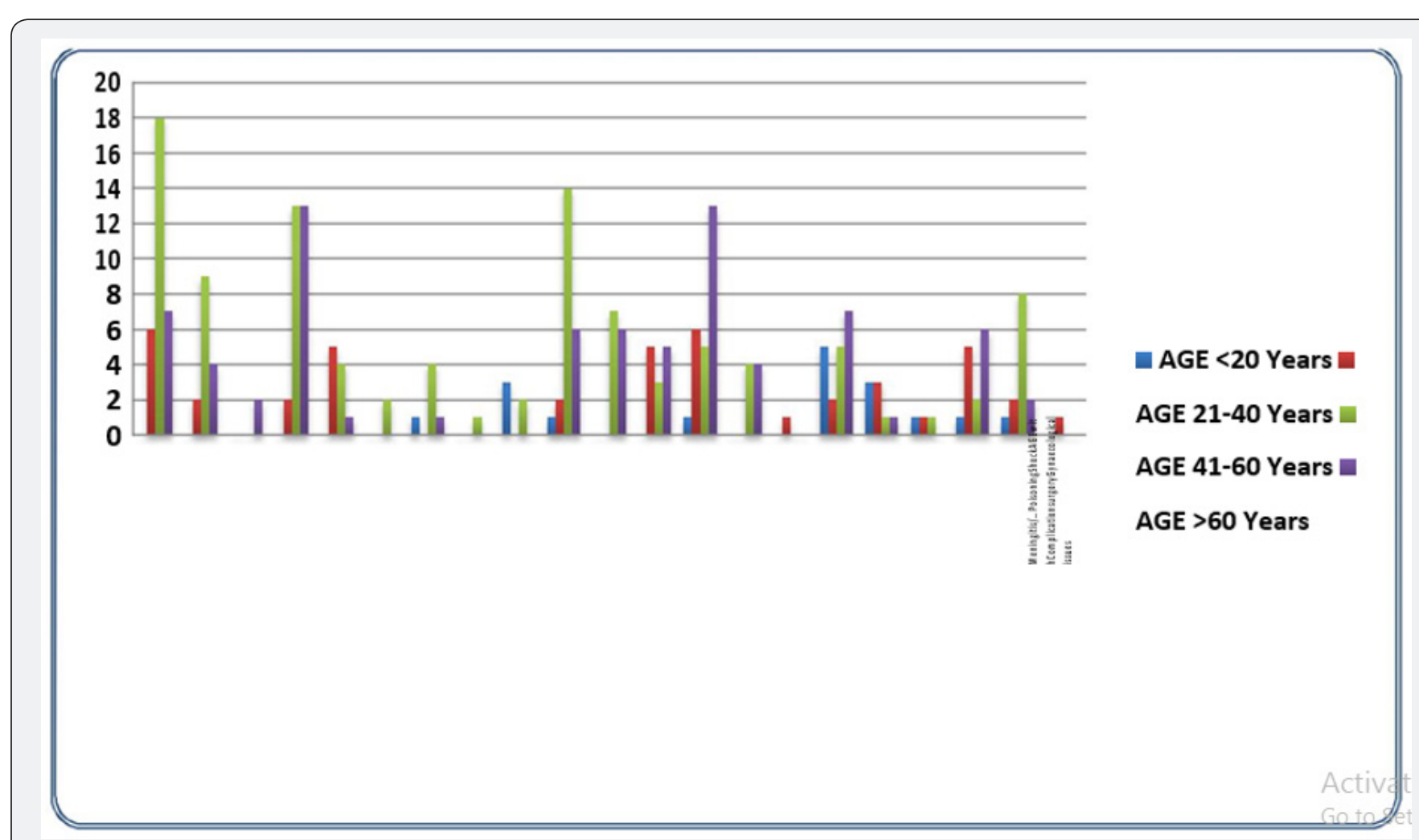

Figure 1: Disease distribution according to Age group.

All the patients in the unit were treated according the written standard operating protocol. Relevant investigations were sent. And it was found to be as Hepatic diseases $12.9 \%$, CCF andPulmonary edema 6 Cellulitis $0.8 \%$, CVA $11.6 \%$, Hypertensive Crisis $4.1 \%$, Arrhythmias $0.8 \%$, Diabetes with complication $2.5 \%$, Carcinoma $0.4 \%, 2.1 \% \mathrm{~Tb}$ with complication, Respiratory issues 9.5\%, ACS 5.4\%, Kidney diseases 5.4\%, Sepsis 10.4\%, GI Bleed
3.3\%, Heat stroke $0.4 \%$, CNS Infection7.9\%, Poisoning 3.3\%, Shock $1.2 \%$, AGE with complication 5.8\%, Surgical and Gynecological issues were $5.4 \%$ and $0.4 \%$. Out of 241 admitted adult 39 (\%), died $16(\%)$, left against medical advice $146(\%)$ were improved and subsequently discharged. Forty (\%) become better and shifted to ward and __ (\%) received mechanical ventilation (Figure 2). 


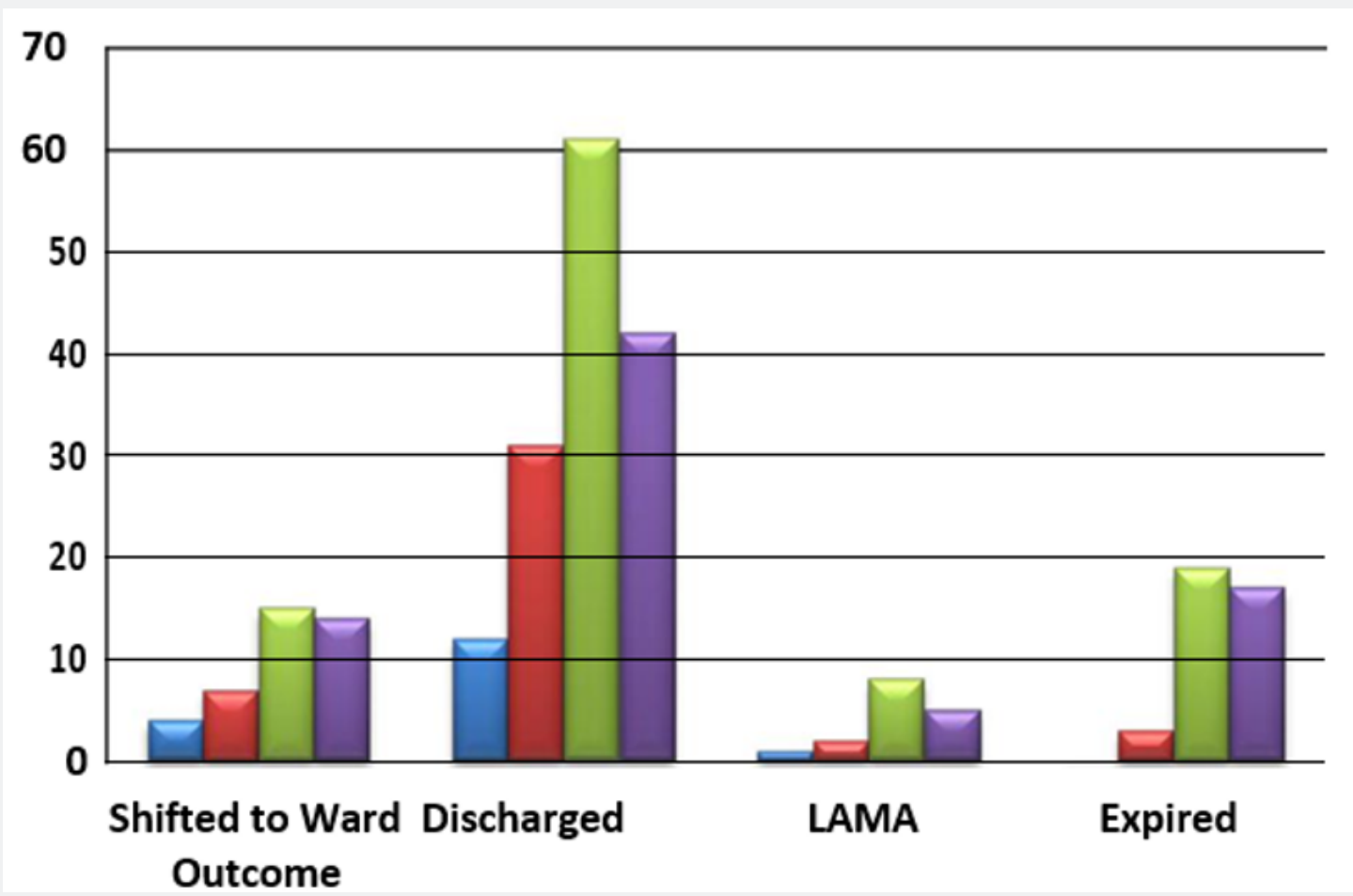

Age $<2$

age 21

$\square$ Age 41

Age $>6$

Figure 2: Age Association with Patient's Outcome.

\section{Result}

The number of admissions in the past over six months in the hospital was 241 in total. Out of which 51\% were males and 49\% female. We divided the patients into four age groups. Group A included less than 20 years and were 7\%, Group B 18\% with age range of 21-40 years, followed by Group $C$ included the age group of the 41-60 years with 43\% and Group D more than 60 years and with $32 \%$. The mean average of the middle age was found more in the intensive unit, as well the prognosis was also found to be better in middle aged patients.

\section{Discussion}

Critical care unit is the main sets up of the hospital, where the severely sick patients get admitted. It need more attention as the disease severity is more in this unit. Patients from emergency ward and from other hospital from tertiary care being shifted and admitted for the better management. In the ICU of our hospital average 400 to 600 patients admit annually. The ratio is high as compare to the local population in tertiary care. Patients admitted with almost young to middle and older age group of both sex with different co morbid ranges from hepatic to renal and pulmonary cases. The cases admitted into the intensive care unit including the liver disease, heart failure, cerebrovascular accident, diabetic complications, heat stroke, diarrhea, pregnancy induced hypertension. The sequence of the cases along with percentage of diseases are as followed. The number of cases with percentage of hepatic disease is on rise among all admissions. The second most is the neurological insult cerebra-vascular disease, followed by septic shock and respiratory infections along with congestive cardiac failure is the 3 most leading cause in our study. The number of cases with percentage of Hepatic diseases 12.9\%, CCF and Pulmonary edema $6.2 \%$, Cellulitis $0.8 \%$, CVA $11.6 \%$, Hypertensive Crisis $4.1 \%$, Arrhythmias $0.8 \%$, Diabetes with complication $2.5 \%$, Carcinoma $0.4 \%, 2.1 \%$ Tb with complication, Respiratory issues 9.5\%, ACS 5.4\%, Kidney diseases 5.4\%, Sepsis 10.4\%, GI Bleed $3.3 \%$, Heat stroke $0.4 \%$, CNS Infection7.9\%, Poisoning 3.3\%, Shock $1.2 \%$, AGE with complication 5.8\%, Surgical and Gynecological issues were $5.4 \%$ and $0.4 \%$ (related to medical issues).

Patients coming from emergency mostly and from outpatient department. Previous studies have recommended that early acknowledgment and intercession at the right time of patients in general wards is probably going to decrease their requirement for critical care, also have suggested development of timely "outreach" administrations from critical care internist to help ward staff in overseeing patients in risk. Our ICU is general and take both medical and surgical patients with a scope of basic sicknesses, medical patients compile the most proportion therefore we have conducted our study in medical patients. In our study the age group of mainly the middle age group of 41 to 60 years old, having good prognosis. As the aim was early recovery, and it's seen by early discharge of the number of the patients with this age group. The prompt diagnosis and timely management and shifting the patients to step down from intensive care unit provides early recovery and better out comes. Protocols regarding intensive care should be followed for the management and step down the numbers of infection. The ultimate goal to reduce the number 
of mortality and morbidity. Surprisingly, middle aged people are found more prone to infections and rate of early recovery was also seen. Hepatic diseases was higher in percent followed by CCF and Pulmonary edema cases. Infections are more source of admission. Other were hypertensive urgency and diabetic crisis.

\section{Conclusion}

The sequence of admissions pattern in the ICU setup with the age group including all from young to older age group. Most number of admissions in Intensive care Unit were of middle age group of both genders with equal distribution and were found to have good prognosis who were shifted timely to step down (to Wards or discharge).

\section{References}

1. Eugenia Cronin, Mick Nielsen, Martin Spollen, Nigel Edwards (2007) Adult Critical Care 8: 40 .

2. Srinivas Murthy, Hannah Wunsch (2012) Clinical review: International comparisons in critical care - lessons learned. Critical Care 16: 218.

3. Simon Finfer, Jean Louis Vincent (2013) Critical Care - An AllEncompassing Specialty. N Engl J Med 369: 669-670.
4. National Center for Biotechnology Information, US National Library of Medicine.

5. Gauri S Shah, Basant K Shah, Anil Thapa, Lokraj Shah, Mishra OP (2011) Admission Patterns and Outcome 2 in a Pediatric Intensive Care Unit in Nepal. British Journal of Medicine and Medical Research 4(30): 21-31.

6. Adel Hassan A Ghoneim, Rabie M Hussein, Reda El Ghamry, Lamiaa Y Mahmoudb (2013) Patterns of admitted cases to Respiratory Intensive Care Unit at Zagazig University Hospitals Egypt. Egyptian Journal of Chest Diseases and Tuberculosis 62(4): 661-668.

7. Rincon F, Mayer SA, Rivolta J, Stillman J, Boden Albala B, et al. (2010) Impact of delayed transfer of critically ill stroke patients from the Emergency Department to the Neuro-ICU. Neurocrit Care13(1): 75-81.

8. Oke DA (2001) Medical admission into the Intensive Care Unit (ICU) of the Lagos University Teaching Hospital. Niger Postgrad Med J 8(4): 179-182.

9. Hendry R Sawe, Juma A Mfinanga, Salum J Lidenge, Boniventura CT Mpondo, Silas Msangi, et al. (2014) Disease patterns and clinical outcomes of patients admitted in intensive care units of tertiary referral hospitals of Tanzania. BMC Int Health Hum Rights 14: 26.

10. De Jonge E, Bosman RJ, Van Der Voort PH, Korsten HH, Scheffer GJ, et al. (2003) Intensive care medicine in the Netherlands, 1997-2001. I. Patient population and treatment outcome. Ned Tijdschr Geneeskd 147(21): 1013-1017.

\section{Your next submission with Juniper Publishers will reach you the below assets}

- Quality Editorial service

- Swift Peer Review

- Reprints availability

- E-prints Service

- Manuscript Podcast for convenient understanding

- Global attainment for your research

- Manuscript accessibility in different formats

( Pdf, E-pub, Full Text, Audio)

- Unceasing customer service

Track the below URL for one-step submission https://juniperpublishers.com/online-submission.php 